\title{
An Experimental Study on the Compressive Dynamic Performance of Polypropylene Fiber Reinforced Concrete for Retaining Structure under Automobile Collision Magnitude
}

\author{
Furong Li, ${ }^{1,2}$ Guoxing Chen $\mathbb{D}^{1},{ }^{1}$ Guizhong $\mathrm{Xu}^{2}{ }^{2}$ and Yongyi $\mathrm{Wu}^{3}$ \\ ${ }^{1}$ Institute of Geotechnical Engineering, Nanjing Tech University, NanJing, JiangSu 210009, China \\ ${ }^{2}$ College of Civil Engineering, Yancheng Institute of Technology, YanCheng, JiangSu 224051, China \\ ${ }^{3}$ Qinghai Research Institute of Investigation \& Design of Water Conservancy \& Hydropower, XiNing 810012, China
}

Correspondence should be addressed to Guoxing Chen; gxchen@njtech.edu.cn

Received 6 May 2020; Revised 14 July 2020; Accepted 15 July 2020; Published 29 July 2020

Academic Editor: Qiang Tang

Copyright (c) 2020 Furong Li et al. This is an open access article distributed under the Creative Commons Attribution License, which permits unrestricted use, distribution, and reproduction in any medium, provided the original work is properly cited.

In order to examine the compressive dynamic performance of polypropylene fiber reinforced concrete to be used for the retaining structure under the automobile collision magnitude, an experimental study was carried out by using hydraulic servo on concrete specimens with 4 different polypropylene fiber contents under 6 loading strain rates. The failure modes and stress-strain curves of concrete under different loading conditions were obtained. Then, by comparatively analyzing the mechanical characteristic parameters of polypropylene fiber reinforced concrete under different loading conditions, the following conclusions are drawn: with the increase of the polypropylene fiber content, the integrity of concrete upon compressive failure is gradually improved. When the polypropylene fiber content is relatively high, the static and dynamic failure modes are basically similar. With the increase of the loading strain rate, the peak compressive stress and elastic modulus of the polypropylene fiber reinforced concrete gradually increase. The increase in the polypropylene fiber content gradually intensifies the effect of loading strain rate on the peak compressive stress dynamic improvement coefficient. The peak strain of polypropylene fiber reinforced concrete is gradually increased with the increase of polypropylene fiber content, while the effect of the loading strain rate on the peak strain shows an obvious discreteness characteristic. Meanwhile, we proposed a relationship equation for describing the peak compressive stress dynamic improvement coefficient based on the coupling effect of the polypropylene fiber content and loading strain rate and further discussed the underlying stress mechanism in detail. Our research findings are of important research significance for the application and promotion of polypropylene fiber reinforced concrete in the engineering practice of retaining structures.

\section{Introduction}

Polypropylene fiber reinforced concrete is a kind of concrete material formed by adding polypropylene fiber into ordinary concrete in accordance with a certain proportion. It is featured with the advantages of high corrosion resistance, strong chemical stability, and high impact resistance. Meanwhile, polypropylene fiber reinforced concrete is able to suppress the generation of early shrinkage cracks to a certain extent so as to improve the ductility of concrete. At present, polypropylene fiber reinforced concrete has been widely concerned and applied in engineering structures, such as dams, retaining walls, and culverts [1-5]. In actual engineering projects, concrete structures are subjected to not only static actions but also dynamic actions including earthquake, collision, and explosion. Thus, there is a great significance to comprehensively study the impact of dynamic performance of concrete [6-9].

Research on the static and dynamic performance of polypropylene fiber reinforced concrete is usually carried out by considering the effects of the size and mix proportion of polypropylene fiber and the influence of loading strain rate [10-12]. Through experimental analysis, Song et al. [13] found that polypropylene fiber could improve the 
compressive strength, tensile strength, and impact resistance of concrete and therefore played a certain role in inhibiting the generation of early shrinkage cracks. Based on an experimental study on the tensile and compressive performance of polypropylene fiber reinforced concrete, Choi et al. [14] found that polypropylene fiber had a greater effect on the tensile strength while a relatively weaker effect on the compressive strength of concrete, and the toughness of concrete was effectively improved by the fiber. Yao [15] conducted an experimental study on the bending performance of polypropylene fiber reinforced concrete. In accordance with the research on the influence of polypropylene fiber content on the strength eigenvalue of concrete, it was found that the compressive strength, tensile strength, and flexural strength of concrete gradually increased with the increase of the fiber content within a reasonable range; however, when the fiber content exceeded a certain proportion, the concrete strength would decrease to a certain extent [16-18]. Regarding the research on the dynamic performance of ordinary concrete, the general conclusions suggested that the peak stress and elastic modulus of ordinary concrete gradually increased with the increase of loading strain rate; meanwhile, the stress dynamic improvement coefficient under tensile loading was higher than that under compressive loading [19-22]. Meanwhile, the influence of concrete strength grade, loading mode, and mix proportion on dynamic rate effect of ordinary concrete were considered. Li [9] carried out experimental research on the dynamic rate effect of ordinary concrete with different strength grades, and Song [6] analyzed the multiaxial dynamic performance of ordinary concrete. The existing research on the dynamic performance of polypropylene fiber reinforced concrete mainly focused on the analysis of the dynamic performance of polypropylene fiber reinforced concrete under ultrahigh strain rate dynamic action. Alhozaimy [23] and Manolis [24] studied the static and dynamic compressive performance of polypropylene fiber reinforced concrete and reported that the polypropylene fiber could improve the static and dynamic compressive strength of concrete. Zhang [25] investigated the dynamic performance of high-content polypropylene fiber reinforced concrete by applying SHPB and concluded that the polypropylene fiber could gradually increase the strain of concrete, which might play a certain role of crack resistance and energy dissipation, but could not improve the compressive strength dynamic improvement coefficient of concrete. Liang et al. [26] examined the effect of ultrahigh strain rate on the compressive performance of polypropylene fiber reinforced concrete and reported that the polypropylene fiber improved the dynamic ultimate toughness of concrete. However, the existing literature on the dynamic performance of polypropylene fiber reinforced concrete under the magnitude of automobile collision and earthquake is very limited. In view of this, a comprehensive study on the dynamic performance of polypropylene fiber reinforced concrete under the effect of medium-low strain rates is of great significance to an in-depth understanding of the safety performance of polypropylene fiber reinforced concrete structures under the magnitude of automobile collision and earthquake.

In this research, we carried out an experimental study on the effect of strain rates at the automobile collision magnitude on the compressive dynamic performance of retaining structures that were made of concrete reinforced with different contents of polypropylene fiber. By applying the hydraulic servo, the failure modes and compression stress-strain curves of polypropylene fiber reinforced concrete under different loading conditions were obtained. Then, by comparatively examining the mechanical performance characteristic parameters under different loading conditions, we comprehensively analyzed the compressive dynamic performance of the polypropylene fiber reinforced concrete from both qualitative and quantitative perspectives and investigated the corresponding stress mechanism in detail.

\section{Experiment Overview}

2.1. Sample Preparation and Design. Our experimental study was mainly carried out on the compressive dynamic performance of concrete with 4 different polypropylene fiber contents. The concrete containing $0 \%$ of polypropylene fiber (i.e., ordinary concrete) was taken as the reference working condition. The designed strength of ordinary concrete mix proportion is $30 \mathrm{MPa}$. The raw materials used for ordinary concrete are as follows: ordinary Portland cement PO 42.5, natural aggregate gravels with particle size ranged from 4 to $16 \mathrm{~mm}$ (coarse aggregates), natural river sands with an apparent density of $2645 \mathrm{~kg} / \mathrm{m}^{3}$, a fineness modulus of 2.6 (fine aggregates), and city tap water. The concrete mix did not contain any additives. In accordance with the "Specification for Mix Proportion Design of Ordinary Concrete" (JGJ55-2011), the mix proportion of ordinary concrete $(0 \%$ fiber) in this study was shown as in Table 1.

The fiber used in this study is polypropylene fiber with physical characteristics as shown in Table 2 (provided by the manufacturer). In accordance with the relevant literature $[5,13-15]$ and the actual applications of retaining structure, we designed 4 different polypropylene fiber contents (i.e., $0 \%, 0.2 \%, 0.4 \%$, and $0.6 \%$ ) and studied their dynamic performance, respectively.

2.2. Experimental Loading Scheme. The effects of different static and dynamic actions are usually realized by the approach of loading strain rate. The relevant literature [6] has summarized the static and dynamic action ranges corresponding to different loading strain rates, as shown in Figure 1. Under different loading strain rates, concrete materials have different effects, which are creep $\left(10^{-8} \sim 10^{-6}\right.$ / $\mathrm{s})$, static load $\left(10^{-6} \sim 10^{-5} / \mathrm{s}\right)$, automobile collision magnitude $\left(5 \times 10^{-5} \sim 5 \times 10^{-3} / \mathrm{s}\right)$, earthquake $\left(10^{-3} \sim 10^{-2} / \mathrm{s}\right)$, impact $\left(1 \sim 10^{2} / \mathrm{s}\right)$, and explosion $\left(10^{2} \sim 10^{3} / \mathrm{s}\right)$ [6]. The low strain rate is $10^{-8} \sim 10^{-5} / \mathrm{s}$, the medium strain rate is $10^{-5} \sim 10^{-1} / \mathrm{s}$, and the high strain rate is higher than $1 / \mathrm{s}$. In this study, we aim to carry out an experimental study on the dynamic 
TABLE 1: Mix proportion (0\% fiber) of ordinary concrete.

\begin{tabular}{lcccc}
\hline Concrete strength grade & & \multicolumn{3}{c}{ Mass of each component per cubic meter/ (kg) } \\
& Cement & Water & Coarse aggregate & Fine aggregate \\
\hline C30 & 461 & 175 & 1252 & 512 \\
\hline
\end{tabular}

TABle 2: Physical properties of polypropylene fiber.

\begin{tabular}{lccccc}
\hline Diameter $(\mathrm{mm})$ & Length $(\mathrm{mm})$ & Tensile strength $(\mathrm{MPa})$ & Elastic modulus $(\mathrm{GPa})$ & Elongation at break $(\%)$ & Density $\left(\mathrm{kg} / \mathrm{m}^{3}\right)$ \\
\hline 0.026 & 19 & 641 & 4.5 & 40 & 0.91 \\
\hline
\end{tabular}

performance of polypropylene fiber reinforced concrete under the magnitude of automobile collision, which corresponds to the loading strain rate ranged from $5 \times 10^{-5} / \mathrm{s}$ to $5 \times 10^{-3} / \mathrm{s}$. Considering the need of comparative analysis with the mechanical performance of polypropylene fiber reinforced concrete under the quasi-static condition, the static loading strain rate was set to be $1 \times 10^{-5} / \mathrm{s}$. Based on all the conditions above, we determined a total of 6 loading strain rates for our experiment: $1 \times 10^{-5} / \mathrm{s}, 5 \times 10^{-5} / \mathrm{s}$, $1 \times 10^{-4} / \mathrm{s}, 5 \times 10^{-4} / \mathrm{s}, 1 \times 10^{-3} / \mathrm{s}$, and $5 \times 10^{-3} / \mathrm{s}$.

The hydraulic servo, as shown in Figure 2, was used to experimentally examine the compressive dynamic performance of concrete with 4 different polypropylene fiber contents under 6 loading strain rates. This hydraulic servo is equipped with an independent load sensor, which collects data for determining the load status of polypropylene fiber reinforced concrete during the loading process. The deformation data was measured by the strain gauge and was used to further determine the load and deformation data during the loading process while satisfying the experimental requirements. By referencing to the relevant literature and the "Standard for Test Method of Mechanical Properties on Ordinary Concrete" (GB/T50081-2016), the dimension of concrete specimen was set to be $100 \mathrm{~mm} \times 100 \mathrm{~mm} \times 100 \mathrm{~mm}$. Considering the randomness and discreteness characteristics of concrete materials, 3 specimens were tested for each working condition, and the mean value was used for analysis. In this paper, $100 \mathrm{~mm} \times 100 \mathrm{~mm} \times 100 \mathrm{~mm}$ cube is used as the dimension of rubber concrete compressive and tensile strength specimens. This size is nonstandard size. In order to facilitate analysis, the compressive peak stress test data are converted according to "the standard for test methods of mechanical properties of ordinary concrete" (GB/T50081-2002), and the compression peak stress reduction factor is 0.95 .

During the experiment, we performed antifriction treatment on the concrete loading surface in order to control the impact of friction between the loading surface of equipment and the compression surface of specimen on the experimental data [3]. Specifically, the combination of threelayer polyethylene plastic film and mechanical butter was used to achieve the purpose of friction control. For the loading method, we adopted the force and displacement mixed control loading mode in the following steps. Firstly, start preloading through force control at a loading rate of $0.3 \mathrm{MPa} / \mathrm{min}$ to gradually increase the compressive stress of concrete from $0 \mathrm{MPa}$ to $3 \mathrm{MPa}$. Secondly, perform unloading

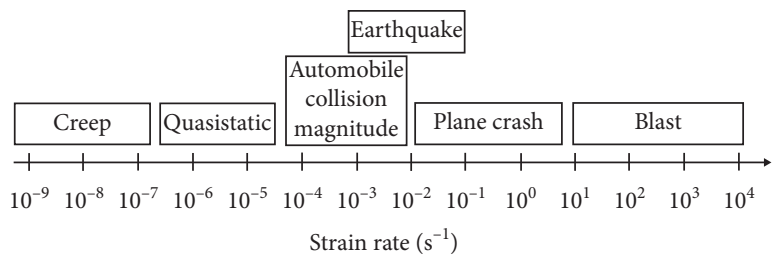

Figure 1: Ranges of strain rate and static/dynamic action.

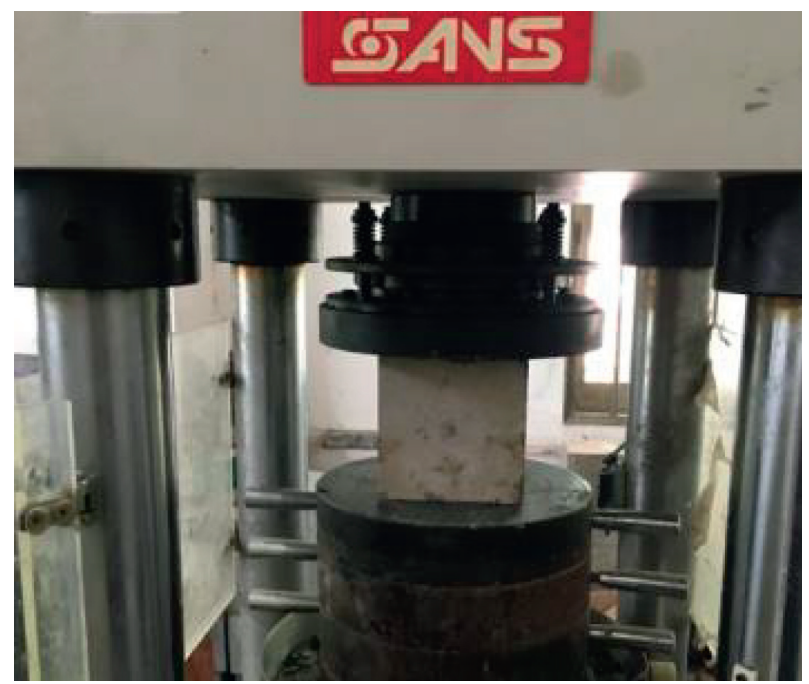

FIGURE 2: Loading equipment.

to reduce the stress to $0 \mathrm{MPa}$, and repeat this process for 3 times in order to control the impact of the gap between the specimen and equipment on the experimental results. Thirdly, adopt displacement control to start the normal loading process (the loading rates were calculated based on the 6 loading strain rates aforementioned). Fourthly, stop loading and data collection after the specimen was damaged.

\section{Analysis of Experimental Results}

3.1. Failure Mode. In accordance with our experimental plan for the dynamic performance of polypropylene fiber reinforced concrete, we obtained the failure modes of concrete with different polypropylene fiber contents under different loading strain rates. Based on the examination of the failure mode, we analyzed the dynamic mechanical performance of the concrete from a macroscopic perspective. In view of 
space limitation, two loading strain rates $\left(1 \times 10^{-5} / \mathrm{s}\right.$ and $5 \times 10^{-3} / \mathrm{s}$ ) were selected for the analysis of each working condition, as shown in Figure 3.

Based on Figure 3, it can be seen that, under the same loading strain rate, the integrity of concrete is gradually improved while the amount and width of cracks gradually decrease upon compressive failure, with the increase of fiber content. The polypropylene fiber reinforced concrete shows two main diagonal cracks with a penetrating development pattern upon compressive failure, and a number of small cracks are distributed around the main diagonal cracks, accompanied by a part of polypropylene fibers that is pulled out or broken. For high strain rate working conditions, the concrete specimens with different polypropylene fiber contents all exhibit a certain level of coarse aggregate damage. The difference in failure mode lies in that the concrete specimens containing $0 \%$ and $0.2 \%$ of polypropylene fiber form uniform cracks on the free surface under the static strain rate working condition, while the failure mode of ordinary concrete mainly exhibits diagonal penetrating cracks under the dynamic loading strain rate. All the concrete specimens containing $0.4 \%$ and $0.6 \%$ of polypropylene fiber exhibit oblique penetrating cracks. A possible explanation is that when the strain rate is relatively high, the time that the concrete is under stress is relatively short so that a uniform stress state cannot be formed in time, leading to incomplete development of cracks; eventually, the failure modes of concrete specimens differ greatly under different strain rates. This failure mode is obviously observed when the polypropylene fiber content is equal to $0 \%$ and $0.2 \%$. When the content of polypropylene fiber is equal to $0.4 \%$ and $0.6 \%$, the concrete failure modes under static or dynamic actions are basically similar. This is mainly because that the intensification of the interaction between the polypropylene fiber and concrete mortar inhibits the development of cracks to a certain extent, and consequently, the specimens are more prone to forming a uniform stress state. In addition, the range of strain rate in this study belongs to a medium-low strain rate state; therefore, when the content of polypropylene fiber is relatively high, the concrete failure modes remain basically similar under static or dynamic strain rate actions.

3.2. Stress-Strain Curve. In accordance with our predetermined compressive loading scheme for different polypropylene fiber contents and loading strain rates, the compressive stress-strain curves of polypropylene fiber reinforced concrete under different loading conditions were obtained, as shown in Figure 4.

In accordance with Figure 4, the development trends of the compressive stress-strain curves for concrete specimens with different polypropylene fiber contents and under different loading strain rates share a strong similarity. Specifically, all the stress-strain curves show good continuity and smoothness. The compressive stress-strain curve can be generally divided into three stages, namely, the elastic stage, the elastoplastic stage, and the descending stage. In the elastic stage, the stress and strain of concrete increase in a linear relationship; in the elastoplastic stage, the stress and strain of concrete increase in a nonlinear relationship; when the stress reaches its peak point, the stress-strain curve will begin to decline, i.e., the stress will gradually decrease as the strain increases further. Based on our preliminary analysis of the compressive stress-strain curves of polypropylene fiber reinforced concrete under different loading conditions, it is found that when the polypropylene fiber content remains the same, the peak compressive stress and elastic modulus of all concrete specimens gradually increase with the increase of the loading strain rate. However, the peak strain is not significantly affected by the loading strain rate. From our analysis, it is obvious that the polypropylene fiber reinforced concrete exhibits a certain level of brittleness failure characteristic upon compressive failure.

\subsection{Eigenvalues of Mechanical Parameters}

3.3.1. Peak Stress. From the compressive stress-strain curves of polypropylene fiber reinforced concrete under different loading conditions, we extracted the peak compressive stress when the loading strain rate is equal to $1 \times 10^{-5} / \mathrm{s}$, in order to examine the effect of polypropylene fiber content on the peak compressive stress under the static loading strain rate, as shown in Figures 5 and 6.

In accordance with Figures 5 and 6, the peak stress of concrete containing $0 \%$ of polypropylene fiber is equal to 25.72 $\mathrm{MPa}$, while the peak stress of concrete containing $0.2 \%, 0.4 \%$, and $0.6 \%$ of polypropylene fiber is equal to 28.83 MPa, 25.27 MPa, and 23.65 MPa, respectively, suggesting an increase of $12.09 \%,-1.76 \%$, and $-8.03 \%$ relative to ordinary concrete. From the overall trend, it is observed that the peak compressive stress increases with the increase of polypropylene fiber content. When the polypropylene fiber content is higher than $0.2 \%$, the peak compressive stress gradually decreases with the increase of polypropylene fiber content. A possible explanation is that the polypropylene fibers, which are evenly distributed in the concrete, have a bonding effect with the cement mortar; when the specimen is subjected to compression, the bonding effect will inhibit the formation and expansion of cracks to a certain extent, making the peak compressive stress of the concrete containing $0.2 \%$ of polypropylene fiber higher than that of ordinary concrete. When the polypropylene fiber content is relatively high, the interface between cement mortar and coarse aggregate will be weakened due to the existence of a large amount of polypropylene fibers. Meanwhile, the workability and fluidity of the concrete mix will gradually decrease, making it difficult for the concrete mix to get compacted. As a consequence, massive defects will be formed inside the concrete. Therefore, the peak compressive stress of concrete containing $0.4 \%$ and $0.6 \%$ of polypropylene fiber is lower than that of ordinary concrete.

In accordance with the analysis above, we proposed a relationship between the proportion of polypropylene fiber under the static loading strain rate $\omega$ and the peak compressive stress variation coefficient $\left(f_{c} / f_{c u}\right)\left(f_{c}\right.$ denotes the 


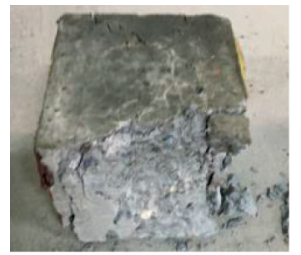

(a)

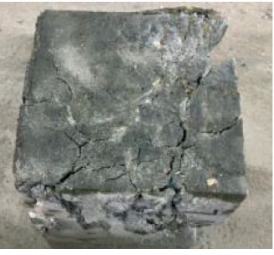

(e)

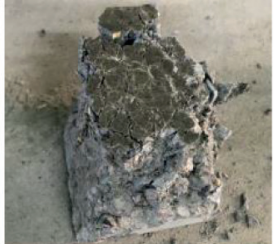

(b)

(I) $0 \%$

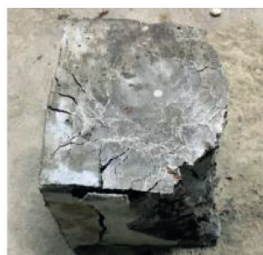

(f)

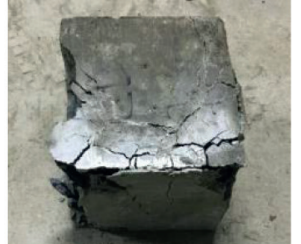

(c)

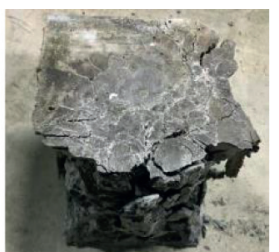

(d)

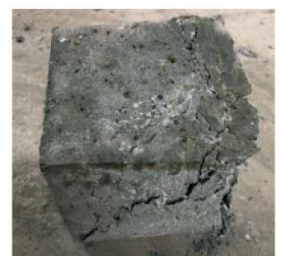

(g)

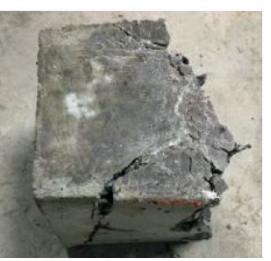

(h)

(III) $0.4 \%$

(IV) $0.6 \%$

FIGURE 3: Compressive failure modes of polypropylene fiber reinforced concrete under different loading conditions. (a) $1 \times 10^{-5} / \mathrm{s}$. (b) $5 \times 10^{-3} / \mathrm{s}$. (c) $1 \times 10^{-5} / \mathrm{s}$. (d) $5 \times 10^{-3} / \mathrm{s}$. (e) $1 \times 10^{-5} / \mathrm{s}$. (f) $5 \times 10^{-3} / \mathrm{s}$. (g) $1 \times 10^{-5} / \mathrm{s}$. (h) $5 \times 10^{-3} / \mathrm{s}$.

peak compressive stress of concrete with various polypropylene fiber contents; $f_{c u}$ denotes the peak compressive stress of ordinary concrete) based on the changing pattern of the relationship between the polypropylene fiber content and the peak compressive stress. Then, by analyzing the experimental data, the relationship equation, as shown in equation (1) and Figure 6, was obtained:

$$
\frac{f_{c}}{f_{c u}}=1+0.5913 \omega-1.2575 \omega^{2} .
$$

Peak stress is an important mechanical parameter for examining the compressive dynamic performance of concrete. We extracted the peak stress points from the compressive stress-strain curves of polypropylene fiber reinforced concrete under different loading conditions in order to comprehensively investigate the influence of polypropylene fiber content and loading strain rate on the compressive performance. The influence of the strain rate effect on the peak stress of concrete can be described quantitatively by the dynamic improvement coefficient $\alpha_{D I \quad F}$, which can be calculated from the following equation [21]:

$$
\alpha_{D I F}=\frac{\sigma_{d}}{\sigma_{s}},
$$

where $\sigma_{d}$ is the peak compressive stress of polypropylene fiber reinforced concrete under static/dynamic loading strain rate (unit: $\mathrm{MPa}$ ) and $\sigma_{s}$ is the peak compressive stress of polypropylene fiber reinforced concrete under static loading strain rate (unit: $\mathrm{MPa}$ ).

Based on the compressive stress-strain curves of polypropylene fiber reinforced concrete under different loading conditions and equation (2), we obtained the relationship between the peak compressive stress and the loading strain rate for the concrete specimens with different polypropylene fiber contents, as shown in Figure 7.
In accordance with Figure 7, when the polypropylene fiber content is equal to $0 \%$, the peak comprehensive stress of concrete under the static loading strain rate of $1 \times 10^{-5} / \mathrm{s}$ is equal to $25.72 \mathrm{MPa}$, while the peak comprehensive stress under the static loading strain rate of $5 \times 10^{-3} / \mathrm{s}$ is equal to $31.63 \mathrm{MPa}$, suggesting a maximum increase of $22.98 \%$ due to the influence of loading strain rate. When the polypropylene fiber content is equal to $0.2 \%, 0.4 \%$, and $0.6 \%$, the peak comprehensive stress of concrete under the static loading strain rate is equal to $37.19 \mathrm{MPa}, 33.61 \mathrm{MPa}$, and $32.16 \mathrm{MPa}$, respectively, suggesting a maximum increase of $28.99 \%$, $33.01 \%$, and $35.98 \%$. By analyzing the general trend, it can be seen that the peak comprehensive stress of concrete with different polypropylene fiber contents gradually increases under the influence of the loading strain rate. With the increase of polypropylene fiber content, the increasing amplitude of the peak compressive stress gradually increases under the influence of the loading strain rate. The existing literature $[6,9,19-21]$ has studied the dynamic performance of ordinary concrete in the medium-low range of strain rate $\left(1 \times 10^{-5} / \mathrm{s} \sim 5 \times 10^{-3} / \mathrm{s}\right)$, and it is found that the peak compressive stress dynamic improvement amplitude is ranged from $20 \%$ to $25 \%$. In our study, the experimental results of the peak compressive stress dynamic improvement amplitude of ordinary concrete are basically consistent with that of the existing literature (difference within $\pm 5 \%$ ). Thus, it can be concluded that the increasing amplitude of the peak compressive stress of polypropylene fiber reinforced concrete under the influence of loading strain rate is higher than that of ordinary concrete. This is because the increase in the strain rate produces a certain level of hysteresis effect on the concrete damage so that the stress of concrete cannot be evenly distributed, resulting in rapid development of cracks. More specifically, damage of a part of coarse aggregate can be observed from the failure mode, which eventually leads to the gradual increase of peak compressive stress. Meanwhile, 


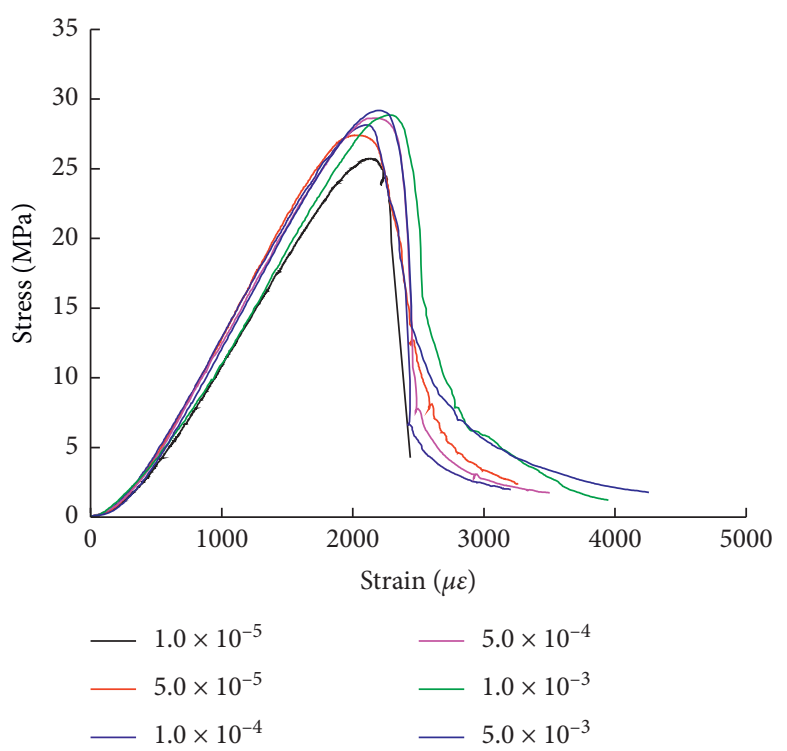

(a)

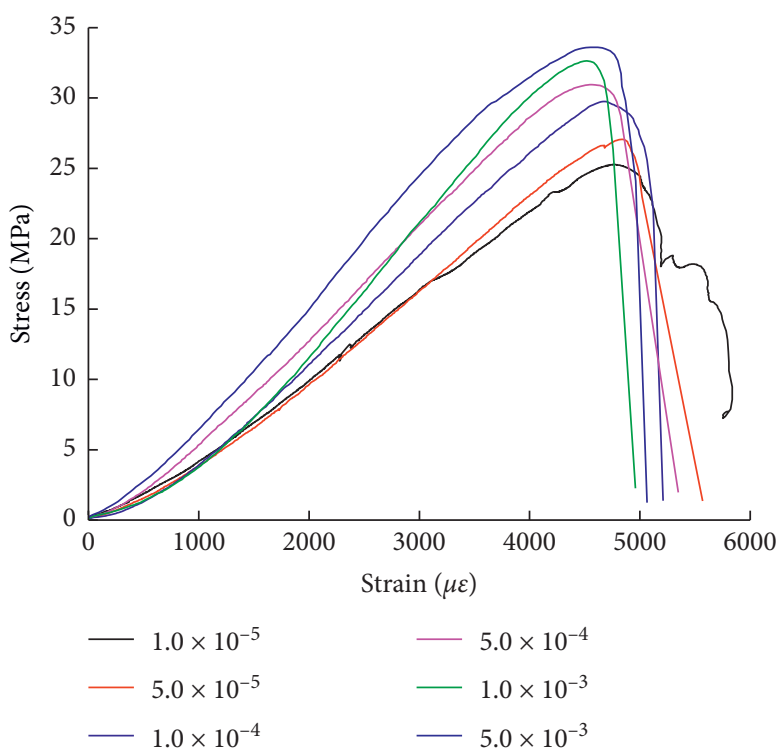

(c)

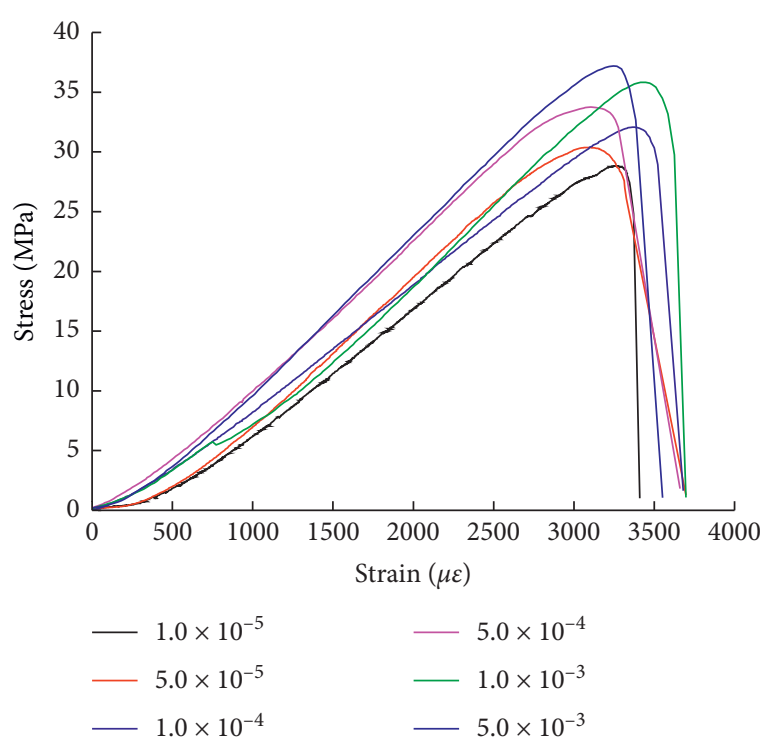

(b)

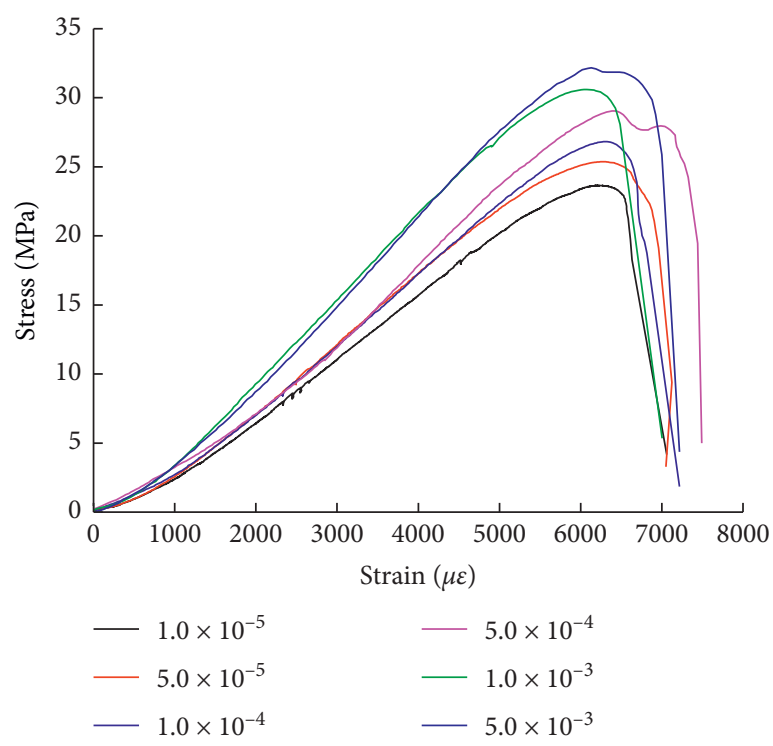

(d)

FIGURE 4: Compressive stress-strain curves of polypropylene fiber reinforced concrete under different loading conditions. (a) $0 \%$. (b) $0.2 \%$. (c) $0.4 \%$. (d) $0.6 \%$.

the increase of polypropylene fiber content will intensify the impact on the lateral deformation of concrete under the compressive action; the higher the strain rate, the greater the impact on lateral deformation. At last, the increase in polypropylene fiber content will increase the increasing amplitude of the peak compressive stress under the influence of the loading strain rate.

The quantitative relationship between the peak compressive stress dynamic improvement coefficient and the loading strain rate for ordinary concrete under medium-low strain rates can be generally described by the equation as shown in equation (3) [27]. In accordance with the qualitative analysis results above, the relationship between the peak compressive stress dynamic improvement coefficient and the loading strain rate for polypropylene fiber reinforced concrete can be described by the same equation:

$$
\alpha_{D I \quad F}=b+a \lg \left(\frac{\dot{\varepsilon_{d}}}{\dot{\varepsilon_{s}}}\right),
$$

where $\dot{\varepsilon}_{d}$ is the compressive static/dynamic loading strain rate of polypropylene fiber reinforced concrete; $\dot{\varepsilon}_{s}$ is the compressive static loading strain rate of polypropylene fiber reinforced concrete; and $a$ and $b$ are coefficients to be determined. Specifically, parameter $b$ is the peak compressive stress improvement coefficient of concrete under the static loading strain rate, which is generally taken as 1 .

In accordance with our mathematical regression analysis based on the experimental data and equation (3), we 


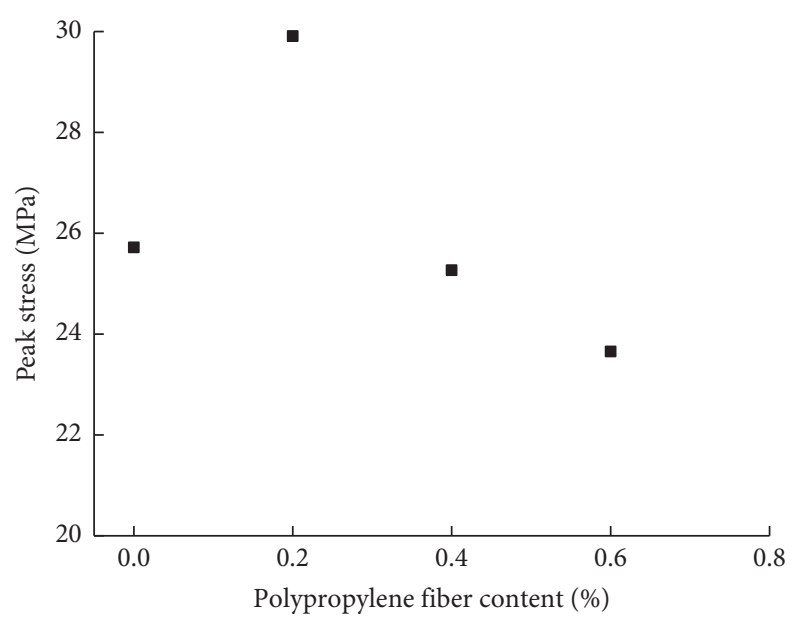

FIGURE 5: Relationship between polypropylene fiber content and peak stress.

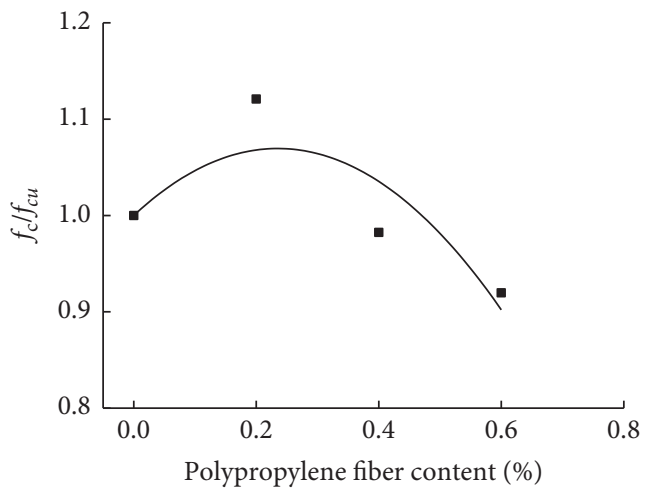

FIGURE 6: Relationship between polypropylene fiber content and stress variation coefficient.

obtained the relationship between the peak compressive stress dynamic improvement coefficient and the loading strain rate for concrete with different polypropylene fiber content, as shown in equations (4)-(7):

$$
\begin{aligned}
& \omega=0 . \%: \alpha_{D I} \quad F=1+0.0835 \lg \left(\frac{\dot{\varepsilon_{d}}}{\dot{\varepsilon}_{s}}\right), \\
& \omega=0.2 \%: \alpha_{D I} \quad F=1+0.1091 \lg \left(\frac{\dot{\varepsilon_{d}}}{\dot{\varepsilon_{s}}}\right), \\
& \omega=0.4 \%: \alpha_{D I} \quad F=1+0.1330 \lg \left(\frac{\dot{\varepsilon_{d}}}{\dot{\dot{\varepsilon}_{s}}}\right), \\
& \omega=0.6 \%: \alpha_{D I} \quad F=1+0.136 \lg \left(\frac{\dot{\varepsilon_{d}}}{\dot{\varepsilon}_{s}}\right),
\end{aligned}
$$

In accordance with our analysis from equations (4)-(7), equation (3) has good applicability to describing the relationship between the peak compressive stress dynamic improvement coefficient and the loading strain rate for concrete with different polypropylene fiber contents. By comparatively analyzing parameter $a$, it is found that $a$ gradually increases with the increase of polypropylene fiber content. Then, based on quantitative analysis, it can be seen that the increase in polypropylene fiber content gradually increases the increasing amplitude of the peak compressive stress of concrete under the influence of the loading strain rate.

In order to put forward the equation for describing the coupling effect of polypropylene fiber content and loading strain rate on the peak compression stress dynamic improvement coefficient, we established a linear equation between the polypropylene fiber content and the slope of equation (3) $a$ based on the qualitative analysis results, as shown in Figure 8 and equation (8).

Then, we expanded parameter $b$ in equation (3) by taking into account the polypropylene fiber content, that is, parameter $b$ is the ratio between the peak compressive stress of concrete with different polypropylene fiber contents $\left(f_{c}\right)$ and the peak compressive stress of ordinary concrete $\left(f_{c u}\right)$. By substituting equations (1) and (8) into equation (3), we thereby established the equation for describing the coupling effect of polypropylene fiber content and loading strain rate on the peak compression stress dynamic improvement coefficient: 


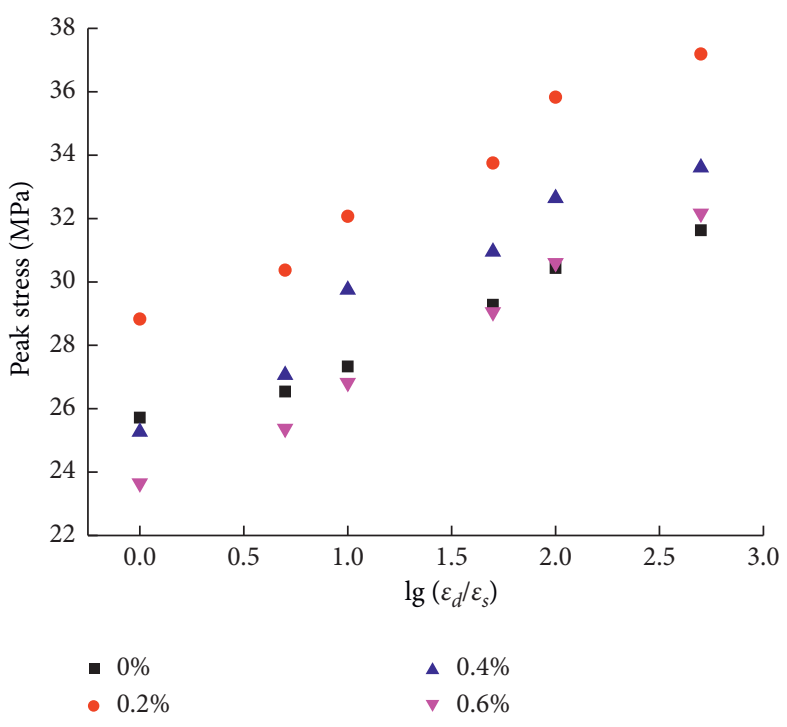

(a)

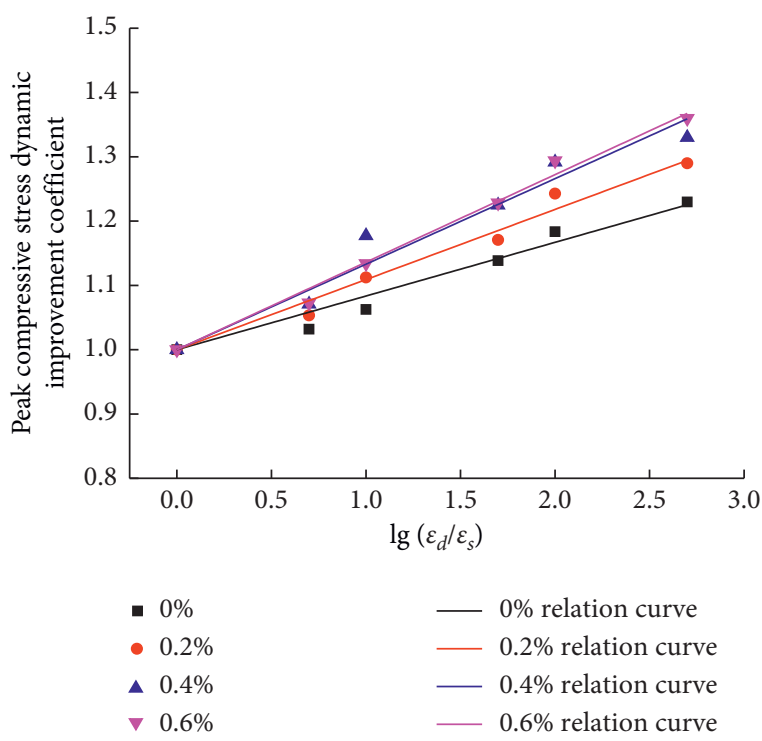

(b)

Figure 7: The compressive peak stress of polypropylene fiber reinforced concrete under different loading conditions. (a) Peak compressive stress. (b) Peak compressive stress dynamic improvement coefficient.

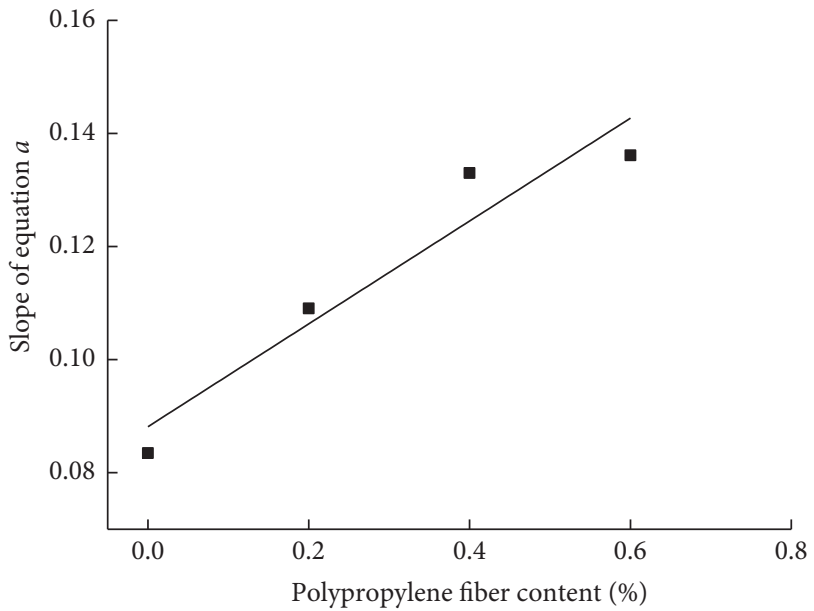

FIGURE 8: Relationship between the polypropylene fiber content and the slope of dynamic improvement coefficient equation a.

$$
a=0.0881+0.0909 \omega \text {. }
$$

$\alpha_{D I} \quad F=\left(1+0.5913 \omega-1.2575 \omega^{2}\right)+(0.0881+0.0909 \omega) \lg \left(\frac{\dot{\varepsilon_{d}}}{\dot{\dot{\varepsilon}_{s}}}\right)$.

3.3.2. Elastic Modulus. The coupling effect of loading strain rate and polypropylene fiber content not only affects the peak stress parameters but also significantly affects the deformation parameters of concrete. Both the elastic modulus and peak strain reflect the deformation parameters of concrete. In order to quantitatively describe the influence of the loading strain rate and polypropylene fiber content on the elastic modulus, this paper defines elastic modulus by equation (10). Meanwhile, the elastic modulus dynamic improvement coefficient $\alpha_{D I} \quad{ }_{F-E}$ is defined by a similar expression as equation (2):

$$
E=\frac{\sigma_{0.5}}{\varepsilon_{0.5}}
$$

where $\sigma_{0.5}$ denotes $50 \%$ of the peak compressive stress of concrete and $\sigma_{0.5}$ denotes the strain corresponding to $50 \%$ of the peak stress of concrete.

In accordance with the compressive stress-strain curves of polypropylene fiber reinforced concrete and equation (10), the elastic modulus and the elastic modulus dynamic improvement coefficient under different loading conditions were obtained, as shown in Figure 9.

From Figure 9, it is found that when the polypropylene fiber content is $0 \%$, the corresponding elastic modulus is equal to $13.54 \times 103 \mathrm{MPa}$ under the loading strain rate of $1 \times 10^{-5} / \mathrm{s}$ and is increased to $16.19 \times 10^{3} \mathrm{MPa}$ under the loading strain rate of $5 \times 10^{-3} / \mathrm{s}$, suggesting a maximum increase of $19.57 \%$ due to the influence of loading strain rate. When the polypropylene fiber content is $0.2 \%, 0.4 \%$, and $0.6 \%$, the corresponding elastic modulus is equal to $9.94 \times 10^{3} \mathrm{MPa}, 5.95 \times 10^{3} \mathrm{MPa}$, and $4.27 \times 10^{3}$, respectively, under static strain rate, and is equal to $12.89 \times 10^{3} \mathrm{MPa}$, $8.23 \times 10^{3} \mathrm{MPa}$, and $5.91 \times 10^{3} \mathrm{MPa}$, respectively, under the loading strain rate of $5 \times 10^{-2} / \mathrm{s}$; thus, the elastic modulus achieves a maximum increase of $29.72 \%, 38.25 \%$, and $38.50 \%$, respectively, due to the influence of the loading strain rate. Considering the range of strain rate from $1 \times 10^{-5} / \mathrm{s}$ to $5 \times 10^{-3} / \mathrm{s}$, the dynamic performance of ordinary concrete under compression was studied in the literature $[20,22]$. The elastic modulus of ordinary concrete is 


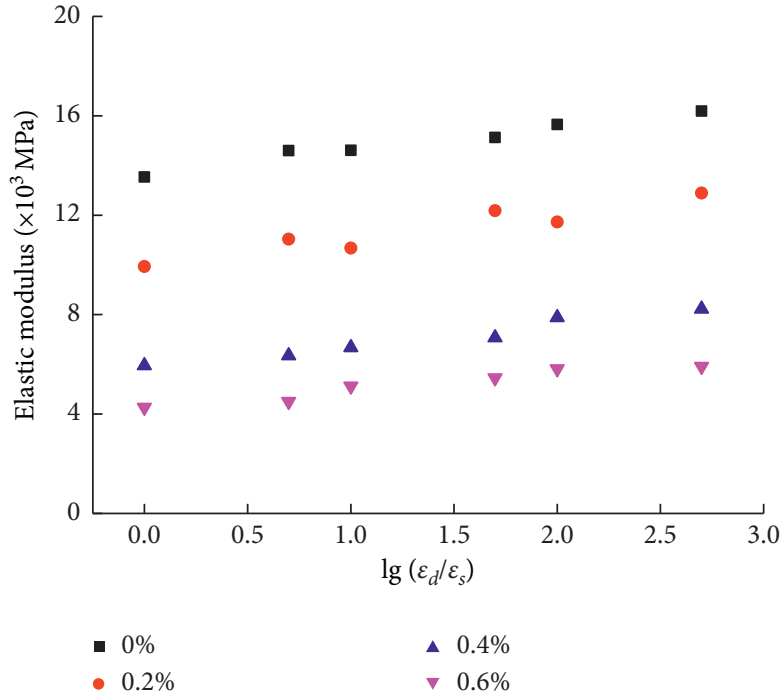

(a)

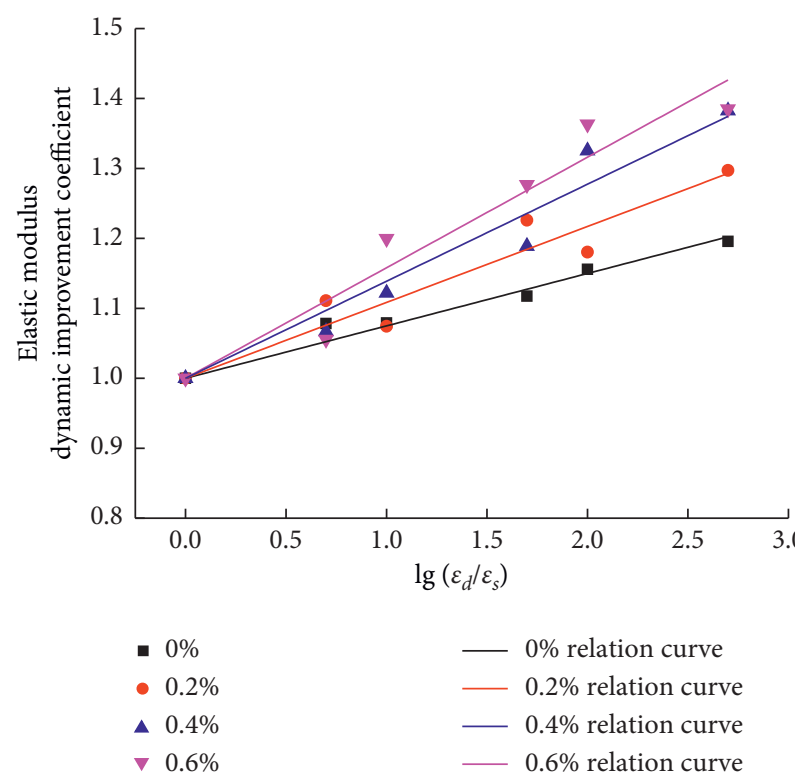

(b)

Figure 9: Elastic modulus of polypropylene fiber reinforced concrete under different loading conditions. (a) Elastic Modulus. (b) Elastic Modulus dynamic improvement coefficient.

increased by $25 \%$ 35\% under the influence of strain rate. The increase range of elastic modulus of ordinary concrete affected by the loading strain rate in this paper is basically consistent with that in this region. From the analysis of the overall trend, it can be seen that, for the same polypropylene fiber content, the elastic modulus gradually increases with the increase of the loading strain rate; with the increase of polypropylene fiber content, the elastic modulus dynamic improvement coefficient is gradually increased under the influence of the loading strain rate. The elastic modulus of polypropylene fiber reinforced concrete shows a rapid increasing trend under the influence of the loading strain rate. This is mainly attributed to the composition of polypropylene fiber in the concrete mixture.

A similar expression as equation (3) was proposed to quantitatively describe the influence of the loading strain rate on the concrete elastic modulus. Based on the quantitative calculation and analysis of the elastic modulus of polypropylene fiber reinforced concrete under different loading conditions, the expressions, as shown in equations (11)-(14) and Figure 9(b), were obtained:

$$
\begin{aligned}
& \omega=0 . \%: \alpha_{D I} \quad F-E=1+0.0749 \lg \left(\frac{\dot{\varepsilon}_{d}}{\dot{\varepsilon}_{s}}\right), \\
& \omega=0.2 \%: \alpha_{D I} \quad F-E=1+0.1085 \lg \left(\frac{\dot{\varepsilon_{d}}}{\dot{\varepsilon_{s}}}\right), \\
& \omega=0.4 \%: \alpha_{D I} \quad F-E=1+0.1387 \lg \left(\frac{\dot{\varepsilon_{d}}}{\dot{\varepsilon}_{s}}\right), \\
& \omega=0.6 \%: \alpha_{D I} \quad F-E=1+0.1580 \lg \left(\frac{\dot{\dot{\varepsilon}_{d}}}{\dot{\dot{\varepsilon}_{s}}}\right) .
\end{aligned}
$$

In accordance with equations (11)-(14) and Figure 9(b), the equation describing the influence of the loading strain rate on the elastic modulus has good applicability to concrete with different polypropylene fiber contents. By comparatively analyzing the slope coefficients in equations (11)-(14), it can be seen that the elastic modulus dynamic improvement coefficient gradually increases with the increase of polypropylene fiber content under the influence of the loading strain rate.

3.3.3. Peak Strain. For the changing pattern of the peak strain of ordinary concrete under the influence of the loading strain rate, the findings of existing literature [19-21] are contradictory: some studies reported that the peak strain of concrete gradually increased with the increase of loading strain rate; some claimed that the peak strain did not change with the loading strain rate; and some believed that the peak strain concrete gradually decreased with the increase of loading strain rate. In accordance with the stress-strain curves of polypropylene fiber reinforced concrete under different loading conditions, we extracted the peak strain values and applied the equation describing the effect of loading strain rate on the peak stress (similar to equation (2)) to examine the effect of loading strain rate on the peak strain of concrete. Specifically, the peak compressive strain of polypropylene fiber reinforced concrete under different loading conditions was obtained, as shown in equation (10).

In accordance with Figure 10, when the polypropylene fiber content is $0 \%$, the peak strain of concrete corresponding to a low loading strain rate ranged from $1 \times 10^{-5} / \mathrm{s}$ to $5 \times 10^{-3} / \mathrm{s}$ falls in the range of $2039 \mu \varepsilon \sim 2282 \mu \varepsilon$, and the peak strain of concrete varies from $-4.50 \%$ to $6.89 \%$ under the influence of the loading strain rate. When the polypropylene fiber content is $0.2 \%, 0.4 \%$, and $0.6 \%$, the peak 


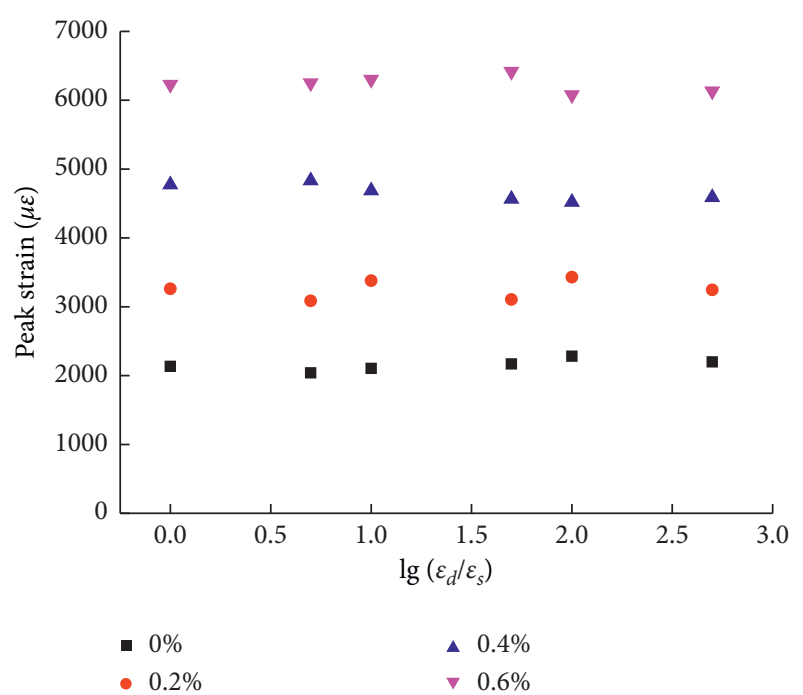

(a)

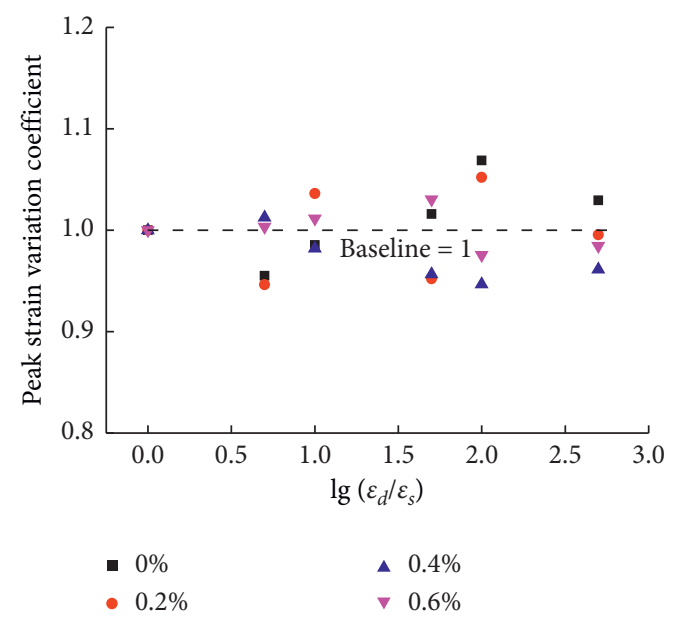

(b)

Figure 10: Peak compressive strain of polypropylene fiber reinforced concrete under different loading conditions. (a) Compressive peak strain. (b) Peak compressive strain variation coefficient.

strain of concrete varies in the range of $3086 \mu \varepsilon \sim 3431 \mu \varepsilon$, $4519 \mu \varepsilon \sim 4833 \mu \varepsilon$, and $6077 \mu \varepsilon \sim 6418 \mu \varepsilon$, respectively, under the influence of the loading strain rate, and the corresponding changing amplitude is in the range of $-5.37 \%$ $5.21 \%,-5.32 \% \sim 1.26 \%$, and $-2.44 \% \sim 3.03 \%$, respectively. Based on the analysis of the results above, it can be seen that the peak strain of concrete gradually increases with the increase of polypropylene fiber content. This is because the polypropylene fibers, which are evenly distributed in the concrete, play a role of a bridge by transferring the load to the uncracked concrete matrix under compression; consequently, the concrete deformation is increased, leading to a significant increase in the ductile deformation capacity of concrete. In this paper, the influence of loading strain rate on the peak compressive strain of concrete with different polypropylene fiber contents exhibits a discrete pattern. This is mainly attributed to the significant randomness and discreteness characteristics of concrete. These two characteristics are primarily reflected from the deformation parameter. The combined effect of randomness, discreteness, and the strain rate leads to the discrete pattern of the effect of loading strain rate on the peak strain rate of concrete.

\section{Conclusions}

In accordance with our analysis on the influence of the polypropylene fiber content and loading strain rate on the compressive dynamic performance of concrete, the following conclusions are drawn:

(1) For the same loading strain rate, the concrete with higher polypropylene fiber content remains a better integrity upon compressive failure. For the same polypropylene fiber content, the concrete remains a relatively better integrity upon compressive failure under a higher strain rate, accompanied by the damage of a part of coarse aggregate. When the polypropylene fiber content is high, the static and dynamic failure modes of concrete are similar.

(2) For the static loading strain rate, the peak compressive stress of concrete increases first followed by a gradual decrease, with the increase of polypropylene fiber content. For the same polypropylene fiber content, the peak compressive stress of concrete gradually increases with the increase of loading strain rate. As the polypropylene fiber content increases, the increasing amplitude of the peak compressive stress dynamic improvement coefficient is gradually increased under the influence of the loading strain rate.

(3) For the same loading strain rate, the elastic modulus and peak strain of concrete gradually increase with the increase of polypropylene fiber content. For the same polypropylene fiber content, the elastic modulus of concrete gradually increases with the increase of loading strain rate, while the peak strain shows a discrete varying pattern.

(4) Based on the coupling effect of the polypropylene fiber content and loading strain rate, we proposed the relationship equation for the peak stress dynamic improvement coefficient of concrete and established the equation describing the relationship between the elastic modulus dynamic improvement coefficient and the loading strain rate of concrete for the same polypropylene fiber content. Meanwhile, we also analyzed the stress mechanism for the effect of polypropylene fiber content and loading strain rate on the compressive dynamic performance of concrete in detail. 


\section{Data Availability}

The data used to support the findings of this study are available from the corresponding author upon request (e-mail: gxchen@njtech.edu.cn).

\section{Conflicts of Interest}

There are no conflicts of interest.

\section{Acknowledgments}

This work was supported by the Key Laboratory of Failure Mechanism and Safety Control Techniques of Earth-rock Dam of the Ministry of Water Resources (YK319010) and National Natural Science Foundation of China (51708273). The authors gratefully acknowledge the financial support.

\section{References}

[1] X. Liu, X. T. Wu, and H. Wei, "Properties of self-compacting lightweight concrete reinforced with steel and polypropylene fibers," Construction and Building Materials, vol. 226, pp. 388-398, 2019.

[2] Z. Yu, X. Q. Huang, and N. Xiao, "Experimental study and failure criterion analysis of plain concrete under combined compression-shear stress," Construction and Building Materials, vol. 179, pp. 198-206, 2018.

[3] Z. Yu, Q. Huang, F. Li et al., "Experimental study on mechanical properties and failure criteria of self-compacting concrete under biaxial tension-compression," Journal of Materials in Civil Engineering, vol. 31, no. 5, Article ID 04019045, 2019.

[4] P. Di Maida, E. C. Sciancalepore, and F. Bondioli, "Effects of nano-silica treatment on the flexural post cracking behaviour of polypropylene macro-synthetic fibre reinforced concrete," Mechanics Research Communications, vol. 88, pp. 12-18, 2018.

[5] J. D. Rios, H. Cifuentes, C. Leiva et al., "Behavior of highstrength polypropylene fiber-reinforced self-compacting concrete exposed to high temperatures," Journal of Materials in Civil Engineering, vol. 30, no. 11, Article ID 04018271, 2018.

[6] S. Shang and Y. Song, "Dynamic biaxial tensile-compressive strength and failure criterion of plain concrete," Construction \& Building Materials, vol. 40, no. 40, pp. 322-329, 2013.

[7] F. Li, Y. Zhang, J. Liu, L. Zhang, and S. Fang, "The dynamical response characteristics of elastic-plastic coal under dynamic load," Journal of Natural Gas Science and Engineering, vol. 29, pp. 497-505, 2016.

[8] X. D. Chen, S. X. Wu, and J. K. Zhou, "Large-beam tests on mechanical behavior of dam concrete under dynamic loading," Journal of Materials in Civil Engineering, vol. 27, no. 10, Article ID 06015001, 2015.

[9] J. Li, X. Yan, and X. Ren, "Large-sample experimental study on uniaxial compressive behavior of concrete under different loading," Journal of Building Structures, vol. 37, no. 8, pp. 66-75, 2016.

[10] H. Korucu, "Polypropylene fiber reinforced concrete plates under fluid impact. Part I: experiments," Structural Engineering and Mechanics, vol. 60, no. 2, pp. 211-223, 2016.

[11] L. Xu, L. Huang, Y. Chi et al., "Tensile behavior of steelpolypropylene hybrid fiber-reinforced concrete," ACI Materials Journal, vol. 113, no. 2, pp. 219-229, 2016.
[12] V. G. Ghorpade and H. S. Rao, "Strength and permeability characteristics of fibre reinforced recycled aggregate concrete with different fibres," Nature Environment and Pollution Technology, vol. 9, no. 1, pp. 179-188, 2010.

[13] P. S. Song, S. Hwang, and B. C. Sheu, "Strength properties of nylon- and polypropylene-fiber-reinforced concretes," $\mathrm{Ce}$ ment and Concrete Research, vol. 35, no. 8, pp. 1546-1550, 2005.

[14] Y. Choi and R. L. Yuan, "Experimental relationship between splitting tensile strength and compressive strength of GFRC and PFRC," Cement and Concrete Research, vol. 35, no. 8, pp. 1587-1591, 2005.

[15] W. Yao, K. J. Li, and K. Wu, "Mechanical properties of hybrid fiber-reinforced concrete at low fiber volume fraction," Cement and Concrete Research, vol. 33, no. 1, pp. 27-30, 2003.

[16] M. Hsie, C. Tu, and P. S. Song, "Mechanical properties of polypropylene hybrid fiber-reinforced concrete," Materials Science \& Engineering A, vol. 494, no. 1-2, pp. 153-157, 2008.

[17] M. H. Beigi, J. Berenjian, A. Sadeghi NikLotfi Omran, and I. M. Nikbin, "An experimental survey on combined effects of fibers and nanosilica on the mechanical, rheological, and durability properties of self-compacting concrete," Materials \& Design, vol. 50, pp. 1019-1029, 2013.

[18] A. Abadel, H. Abbas, T. Almusallam et al., "Mechanical properties of hybrid fibre-reinforced concrete - analytical modelling and experimental behaviour," Magazine of Concrete Research, vol. 68, no. 15-16, pp. 823-843, 2016.

[19] J. B. Menzies and P. R. Sparks, "The effect of rate of loading upon the static and fatigue strengths of plain concrete in compression," Magazine of Concrete Research, vol. 25, no. 83, pp. 73-80, 1973.

[20] P. H. Bischoff and S. H. Perry, "Compressive behaviour of concrete at high strain rates," Materials and Structures, vol. 24, no. 6, pp. 425-450, 1991.

[21] S. Zeng and J. Li, "Experimental study on uniaxial compression behavior of concrete under dynamic loading," Journal of Tongji University (Natural Science), vol. 41, no. 1, pp. 7-10, 2016.

[22] L. Shi, Y. Song, and L. Shen, "Experimental study on uniaxial compression properties of large aggregate and wet-screened concrete at different strain rates," World Earthquake Engineering, vol. 32, no. 2, pp. 270-276, 2016.

[23] A. M. Alhozaimy, P. Soroushian, and F. Mirza, "Mechanical properties of polypropylene fiber reinforced concrete and the effects of pozzolanic materials," Cement and Concrete Composites, vol. 18, no. 2, pp. 85-92, 1996.

[24] G. D. Manolis, P. J. Gareis, A. D. Tsonos, and J. A. Neal, "Dynamic properties of polypropylene fiber-reinforced concrete slabs," Cement and Concrete Composites, vol. 19, no. 4, pp. 341-349, 1997.

[25] H. Zhang, Y. Liu, H. Sun, and S. Wu, "Transient dynamic behavior of polypropylene fiber reinforced mortar under compressive impact loading," Construction and Building Materials, vol. 111, pp. 30-42, 1996.

[26] N. Liang, P. Yang, X. Liu et al., "A study on dynamic compressive mechanical properties of multi-size polypropylene fiber concrete under high strain rate," Materials Review, vol. 32, no. 1, pp. 288-294, 2018.

[27] F. Li, Y. Z. Yu, and Y. Hu, "Experimental study on dynamic performance of self-compacting lightweight Aggregate concrete under compression," Advances in Civil Engineering, vol. 2019, no. 8, pp. 1-8, 2019. 